\title{
PARAGANGLIOMA IN THE SPINE: CASE REPORT
}

Maurício Santos Gusmão', Eduardo Gil França Gomes², Rony Brito Fernandes ${ }^{3}$, Djalma Castro de Amorim Junior², Marcus Thadeu Venâncio Simões ${ }^{4}$, Joilda Fontes Gomes ${ }^{5}$, Jayme Batista Freire ${ }^{6}$, Marcos Almeida Matos ${ }^{7}$, Joseph Azulay ${ }^{8}$

\section{ABSTRACT}

Paragangliomas are rare neuroendocrine tumors that originate in specialized cells derived from neural crest cells, affecting the region of the carotid or jugular-tympanic artery. They are rare within the spinal canal and, when they occur, they are normally found in the extramedullary intradural compartment of the lumbosacral region. This report presents the case of a 26-year-old male patient who was diagnosed with pathological fracture of the thoracic spine (T10), secondary to paraganglioma, thus causing complete spinal cord injury. Surgical treatment was performed at the General Hospital of the State of Bahia and the complication of excessive bleeding occurred, which indicated that there had been a need for prior embolization. Because of this, the authors recommend caution when evaluating and treating this rare lesion that causes neurological deficits, with careful surgical planning in order to catastrophic complications.

Keywords - Paraganglioma; Spinal Neoplasms; Spinal Cord Compression

\section{INTRODUCTION}

Paragangliomas arising in the spinal canal are rare and, when they occur, they are usually found in the extramedullary intradural compartment of the lumbosacral region. It is very rare to find them in the thoracic region, and so far only five cases have been described ${ }^{(1-3)}$.

They were first described by Alfred Kohn, in 1903. These lesions are rare neuroendocrine neoplasms that originate in specialized cells derived from the neural crest. They are extremely vascularized and continuous with tissues that are integrated in autonomic functions. They are most frequent in the carotid or jugulotympanic region (around $80 \%$ to $90 \%$ of the cases), but they may develop at any site where chromaffin cells exist, from the base of the cranium to the bladder ${ }^{(1,2)}$.

Pheochromocytomas are tumors that originate from chromaffin cells, which are specialized non-epithelial cells of the neural crest that are associated with sympathetic ganglia during fetal life. After birth, most of these cells degenerate and the largest numbers of residual chromaffin cells remain in the adrenal medulla ${ }^{(3,4-8)}$.

The aim of this study was to describe a rare case of paraganglioma of the thoracic spine, and also to review the literature on this topic.

\section{CASE REPORT}

The patient was a 26-year-old married man who was a general services auxiliary born and living in the city of Salvador, in the state of Bahia. He did not present other comorbidities. He sought an orthopedic outpatient clinic in September 2009 with a complaint

1 - Orthopedist and Specialist in Spinal Surgery; Attending Physician at the TRM Service, State General Hospital; Coordinator and Preceptor of Fourth-year Residence relating to the Spine, Hospital of Santa Casa de Misericórdia da Bahia, Salvador, BA, Brazil.

2 - Orthopedist and Specialist in Spinal Surgery; Attending Physician at the TRM Service, State General Hospital; Preceptor of Fourth-year Residence relating to the Spine, Hospital of Santa Casa de Misericórdia da Bahia, Salvador, BA, Brazil.

3 - Fourth-year Resident Physician in Spinal Surgery, State General Hospital and Hospital of Santa Casa de Misericórdia da Bahia, Salvador, BA, Brazil.

4 - Orthopedist and Specialist in Spinal Surgery; Attending Physician at the TRM Service, State General Hospital, Salvador, BA, Brazil.

5 - Orthopedist and Specialist in Spinal Surgery; Attending Physician at the TRM Service, State General Hospital, Salvador, BA, Brazil.

6 - Orthopedist and Specialist in Spinal Surgery; Head of the TRM Service, State General Hospital, Salvador, BA, Brazil.

7 - Orthopedist and Specialist in Pediatric Orthopedics; Adjunct Professor and PhD from the Bahia School of Medicine and Public Health; Coordinator of Medical Residence in Orthopedics and Traumatology, Hospital of Santa Casa da Misericórdia da Bahia, Salvador, BA, Brazil.

8 - Orthopedist and Traumatologist ay the TRM Service, State General Hospital, Salvador, BA, Brazil.

Work performed in the TRM Group, State General Hospital, Salvador, Bahia.

Correspondence: Rony Brito Fernandes, Rua João Bião de Cerqueira 251, ap. 901, bloco A, Bairro Pituba, 41830-580, Salvador, BA. E-mail: ronybritofernandes@hotmail.com Work received for publication: May 23, 2011; accepted for publication: June 6, 2011

The authors declare that there was no conflict of interest in conducting this work 
of insidious diffuse pain in the thoracic region, with progressively diminished muscle strength and paresthesia in the left lower limb. Simple radiographs of the thoracolumbar spine were requested, which from the information gathered, did not present any significant abnormalities. Nevertheless, at this time, the patient was advised to seek a medical service specializing in spinal surgery. In December 2009, the patient reported that he had suffered a fall, from his own height, which evolved with worsening of the thoracic pain and instantaneous loss of muscle strength in the lower limbs, such that he was no longer able to walk.

He was admitted to the State General Hospital (HGE), in Salvador, BA, with a complaint of intense back pain, and in the physical examination he presented pain on palpation at the levels T9-T11. In the neurological examination, he presented a motor and sensory deficit in the lower limbs, muscle strength grade 0 at the level T10, and was classified on the Frankel scale as A. Simple radiography on the thoracic spine in anteroposterior and lateral views showed osteolysis in association with significant loss of height in the vertebral body T10. Magnetic resonance showed destruction and hypersignal at $\mathrm{T} 10$ in the $\mathrm{T} 1$ sequence, and hypersignal in the $\mathrm{T} 2$ sequence, with significant spinal cord compression, causing edema (Figures 1, 2, 3 and 4). The laboratory tests were within the normal ranges. At this time, a diagnosis of pathological fracture of T10 was made, with the etiology still to be clarified.

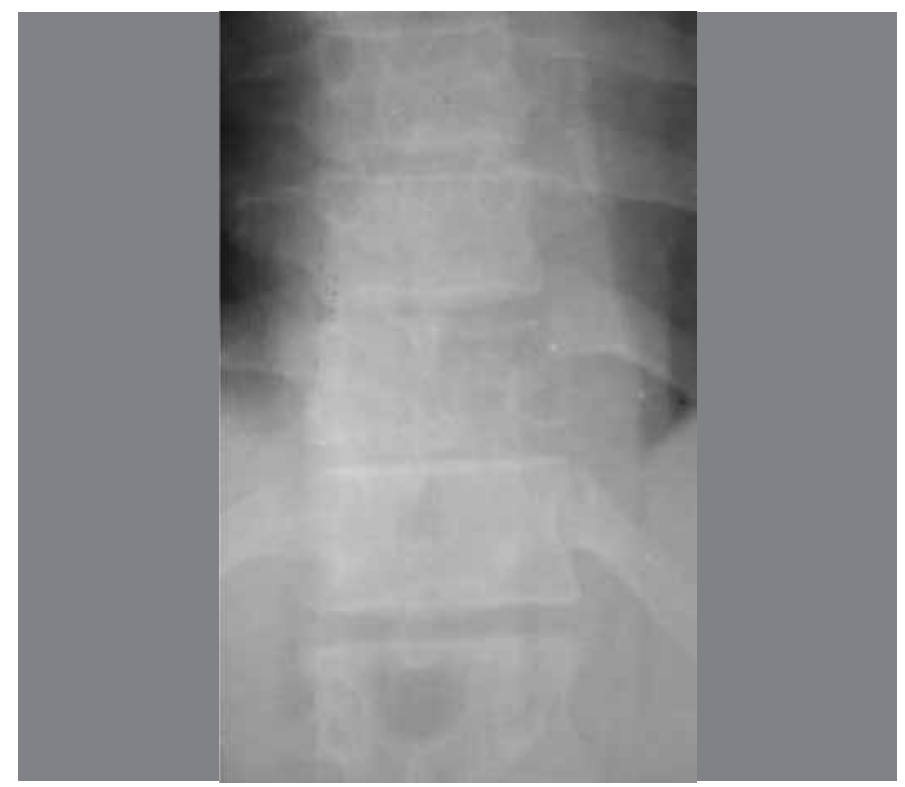

Figure 1 - Radiograph of the thoracolumbar spine (T9-L1) in AP view, showing separation of the pedicles of T10 and osteolysis of the vertebral body.

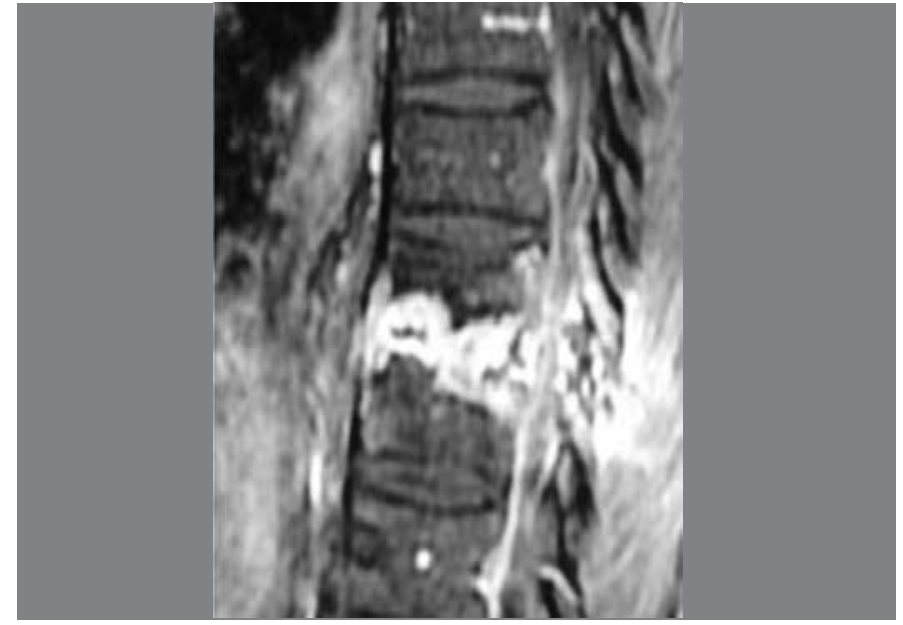

Figure 2 - T1 sagittal MRI showing hypersignal and destruction of the vertebral body.

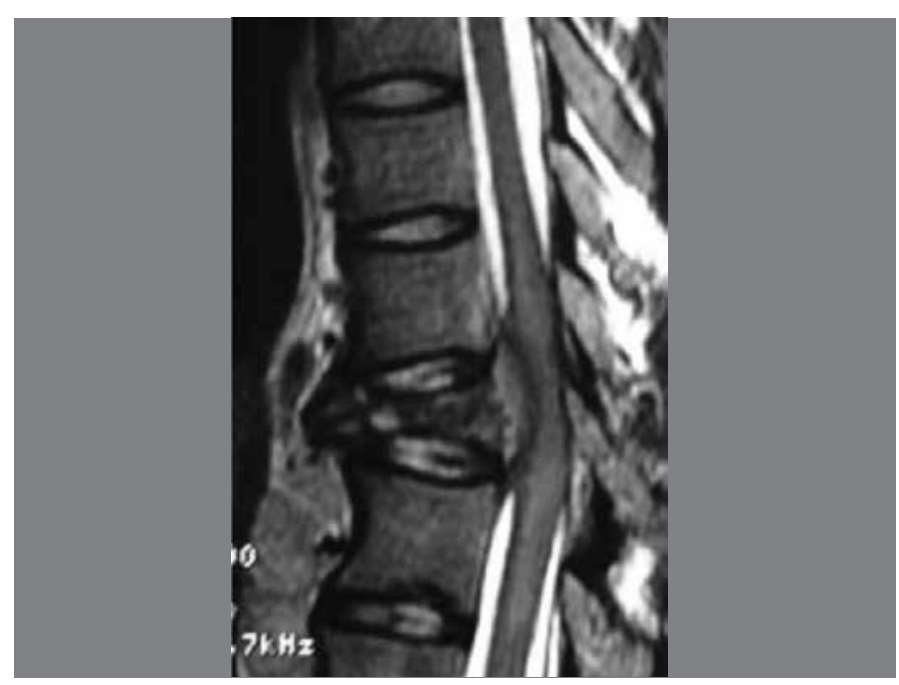

Figure 3 - T2 sagittal MRI showing vertebral compression in the spinal cord and destruction of the vertebral body, while sparing adjacent discs.

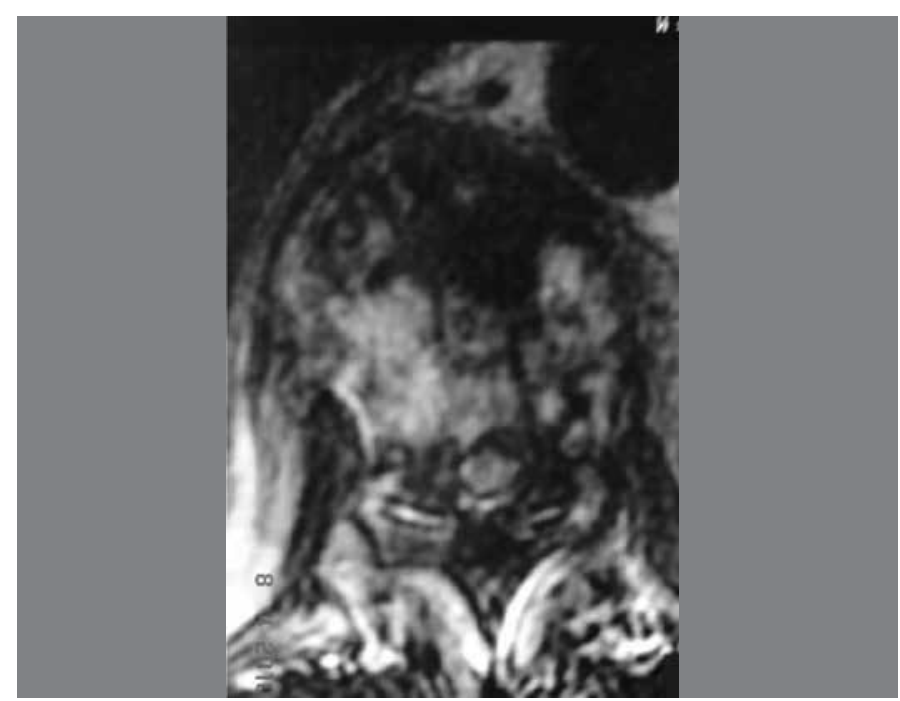

Figure 4 - Sagittal MRI showing central destruction and compression of the spinal canal. 
Surgical treatment of the pathological fracture in T10 was performed on February 5, 2010, with planning of a biopsy, tumor resection with spinal cord decompression, stabilization with arthrodesis and instrumentation by means of posterior route, in T8-T12. However, during the procedure, from the time of the access until placement of the first pedicle screws, it could be seen that the tumor growth was highly vascularized, with hemorrhaging that was difficult to control. Therefore, at this time, because of the hemodynamic risks, the planned surgery was modified, and only careful hemostasis was performed. Material was sent for anatomopathological examination and culturing.

After clinical stabilization, the patient was transferred to the Clinical Hospital of the Federal University of Bahia for arterial embolization of the tumor, to be performed by the vascular surgery team. This procedure was successful.

The culturing was negative, and the anatomopathological examination showed the presence of small cells with irregular rounded nuclei, with intercalated neurons that were organized in large groups, compatible with paraganglioma (Figures 5 and 6).

After this diagnostic clarification, tumor resection in association with decompression and stabilization was planned, to be performed on April 9, 2010. During this procedure, and despite the previous embolization, excessive bleeding occurred, which was difficult to control and obliged the surgical team to abort the resection. All that was achieved was decompression of the spinal cord canal by means of laminectomy, and stabilization by means of arthrodesis via a posterior route at T8-L1. Subsequently, the patient was sent for oncological treatment at another hospital unit (Figures 7 and 8).

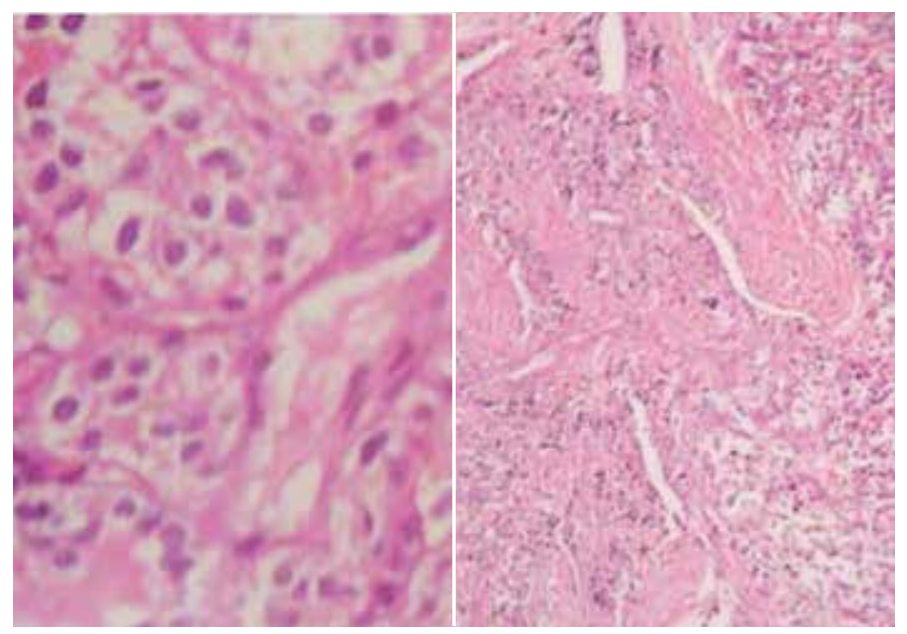

Figures 5 and 6 - Uniform nuclei without mitosis.

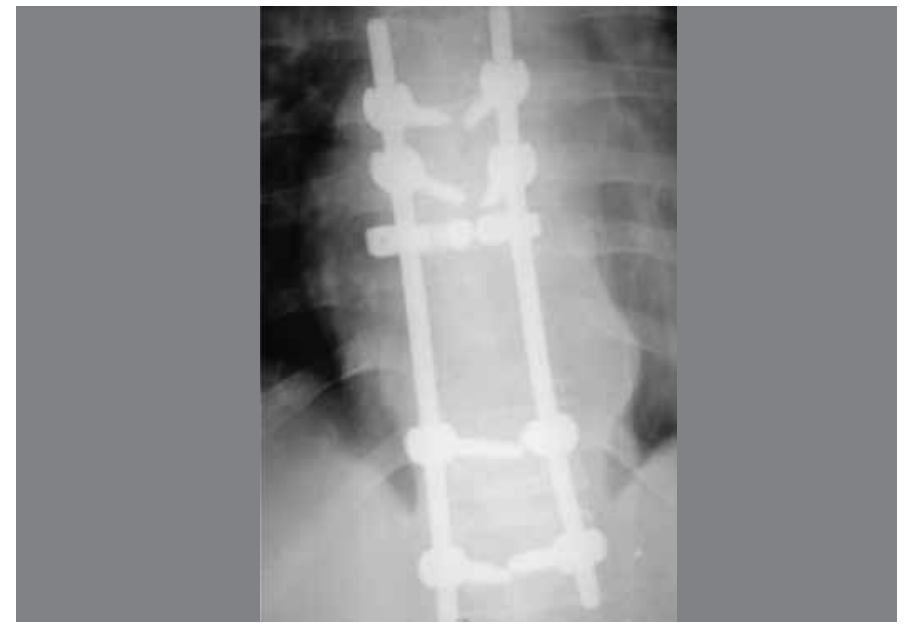

Figure 7 - Radiograph in AP view showing arthrodesis of T8-L1.

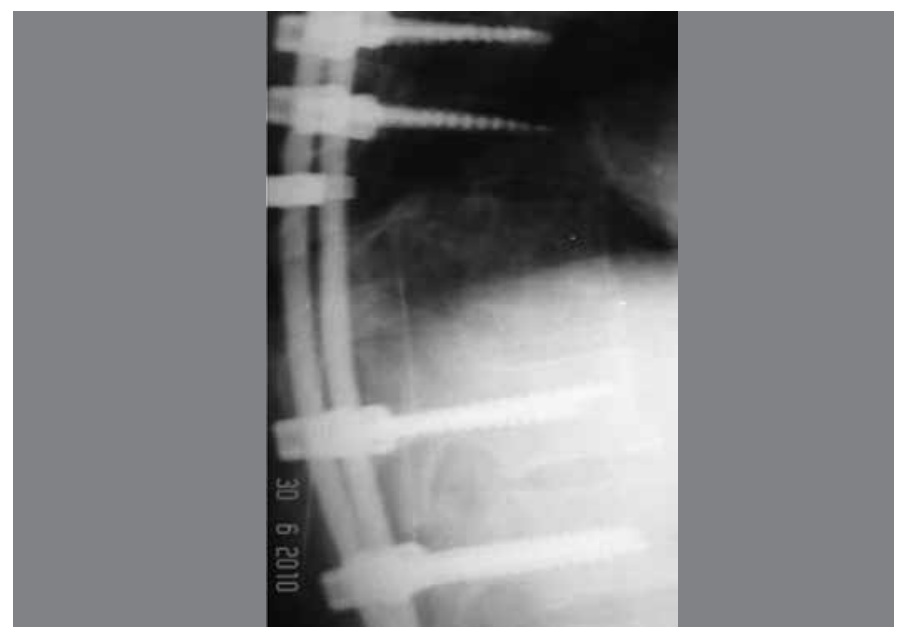

Figure 8 - Radiograph in lateral view showing arthrodesis of T8-L1.

\section{DISCUSSION}

Paragangliomas are rare neuroendocrine tumors that produce catecholamines, and 80 to $90 \%$ of the incidence is in the carotid body and jugular tissue. They are highly vascularized tumors that can affect any part of the body, including the spine. In the spine, the segment most affected is the lumbar region, although the thoracic region may more rarely be affected. Such patients may evolve with pathological fractures and consequent compression of the spinal cord. Most authors recommend preoperative embolization whenever this type of lesion is diagnosed.

There is scientific documentation that around $80 \%$ to $90 \%$ of the cases of paraganglioma arise in the carotid body or jugular vein, although they may occur in other localities such as the thoracic spine, albeit rare in this region. When these tumors occur in the spine, they are mostly intradural and located in the cauda equina, with 80 cases documented in the literature ${ }^{(9,10)}$. 
In the study by Gelabert-González ${ }^{(1)}$ on paraganglioma in the lumbar region, greater incidence among males was demonstrated, with an age range from 9 to 74 years and insidious manifestations of lumbosciatic pain, with spinal cord compression compatible with the case reported here.

In the reports by John et al apud Gelabert-González ${ }^{(1)}$, Constantini et $\mathrm{al}^{(2)}$ and Cruz Ortiz et $\mathrm{al}^{(9)}$, six cases of paraganglioma in the thoracic spine were described, with a clinical condition of spinal cord compression and extradural location. Their description is consistent with our case, in which the tumor started to present insidiously over the course of several months. The significant degree of spinal cord compression in our case indicates that the tumor had already been present for a period of several months, probably with slow growth until the time of the trauma, i.e. similar to the cases presented previously.

Magnetic resonance imaging findings demonstrated destruction of the T10 vertebral body, with hypersignal areas in $\mathrm{T} 2$ that were suggestive of bone edema and spinal cord compression, similar to other published cases $^{(1,3,9)}$.

Paragangliomas are highly vascularized tumors, which also makes it difficult to achieve total surgical resection. Cases of recurrence when not totally resected have been described in the literature. In the case presented here, the procedure was aborted because of the risk to the patient's life due to hypovolemia, following intense hemorrhaging, with technical difficulty in resecting the tumor growth, which was highly vascularized $^{(1,3,9)}$.

This case provides important indications for managing occurrences of paraganglioma. Firstly, it reflects the need for prior embolization of these lesions because of the abundant intraoperative bleeding that may occur. Secondly, it provides a warning that total resection may be impossible in many cases. Finally, it needs to be added that this case draws attention to the need to search for uncommon or even rare lesions whenever surgeons are faced with a condition of neurological deficit in association with pathological fracturing.

\section{REFERENCES}

1. Gelabert-González M. Paragangliomas of the lumbar region. Report of two cases and review of the literature. J Neurosurg Spine. 2005;2(3):354-65.

2. Constantini S, Soffer D, Siegel T, Shalit MN. Paraganglioma of the thoracic spinal cord with cerebrospinal fluid metastasis. Spine (Phila Pa 1976). 1989;14(6):643-5.

3. Fitzgerald LF, Cech DA, Goodman JC. Paraganglioma of the thoracic spinal cord. Clin Neurol Neurosurg. 1996;98(2):183-5.

4. Cybulski GR, Nijensohn E, Brody BA, Meyer PR Jr, Cohen B. Spinal Cord compression from a thoracic paraganglioma: case report. Neurosurgery. 1991;28(2):306-9.

5. Solymosi L, Ferbert A. A case of spinal paraganglioma. Neuroradiology. 1985;27(3):217-9.

6. Lehmen JA, Babbel DM, Mikhitarian K, Choma TJ. Paraganglioma presenting as metastatic lesion in a cervical vertebra: a case report and review of the literature. Spine (Phila Pa 1976). 2010;35(5):E152-4.

7. Shankar GM, Chen L, Kim AH, Ross GL, Folkerth RD, Friedlander RM. Composite ganglioneuroma-paraganglioma of the filum terminale. J Neurosurg Spine. 2010;12(6):709-13

8. Faro SH, Turtz AR, Koenigsberg RA, Mohamed FB, Chen CY, Stein H. Paraganglioma of the cauda equina with associated intramedullary cyst: MR findings. AJNR Am J Neuroradiol. 1997;18(8):1588-90.

9. Cruz Ortiz H, Gutiérrez Díaz Ceballos ME, Rosas Coty MP, Arana Rivera E, Cervantes Zúñiga I. Paragangliomas estudio clinicopatológico de 25 casos. Rev Med Hosp Gen Mex. 1999;62(3):165-71.

10. Houten JK, Babu RP, Miller DC. Thoracic paraganglioma presenting with spinal cord compression and metastases. J Spinal Disord Tech. 2002;15(4):319-23. 